\title{
Photometric study of a pulsating component in the eclipsing binary Y Cam
}

\author{
S.-L. Kim ${ }^{1}$, J. W. Lee ${ }^{2}$, J.-H. Youn ${ }^{1}$, S.-G. Kwon ${ }^{1}$, and C. Kim ${ }^{3}$ \\ ${ }^{1}$ Korea Astronomy Observatory, Daejon, 305-348, Korea \\ 2 Department of Astronomy and Space Science, Chungbuk National University, Cheongju, 361-763, Korea \\ 3 Department of Earth Science Education, Chonbuk National University, Chonju, 561-756, Korea
}

Received 5 April 2002 / Accepted 16 May 2002

\begin{abstract}
We present CCD photometric results of the eclipsing binary Y Cam, whose primary component has been known to be a $\delta$ Scuti type pulsator. Observations were performed for 16 nights, including two primary minima, from November 2000 to May 2001. After correction for light variations caused by the eclipsing phenomenon, we investigated its pulsating features in detail. We derived four pulsation frequencies of $15.0473 \mathrm{c} / \mathrm{d}, 18.2852 \mathrm{c} / \mathrm{d}, 14.8203 \mathrm{c} / \mathrm{d}$ and $17.7348 \mathrm{c} / \mathrm{d}$ using all data except for the primary eclipsing phase. The first frequency turned out to be constant over 40 years but the other frequencies have been changed or newly excited. It should be noted that $V$ amplitude of the first frequency decreased to about a half in comparison with the previous results.
\end{abstract}

Key words. stars: binaries: eclipsing - stars: variables: $\delta$ Sct - stars: individual: Y Cam

\section{Introduction}

$\delta$ Scuti type pulsating components in eclipsing binary systems are very interesting objects which show eclipse and pulsation simultaneously. From an asteroseismological point of view, they are attractive observing targets because they allow precise determination of masses and radii for each component, identification of non-radial modes using the amplitude and phase changes during the eclipse, and detection of sectoral modes, etc. (Mkrtichian et al. 2002a). We can also estimate the evolutionary stage of the mass-accreting component, asynchronization and differential rotation of semi-detached Algol type systems from an asteroseismic approach (Mkrtichian et al. 2002b). Nevertheless, only six eclipsing binary stars were reported in a generalized review on $\delta$ Scuti stars in double and multiple systems by Lampens \& Boffin (2000). So far, AB Cas (Rodríguez et al. 1998) and RZ Cas (Ohshima et al. 2001) have been studied photometrically in detail. This is probably due to difficulties in observing small amplitude oscillations, in comparison with large light variations caused by the eclipsing phenomenon.

The Central Asian Network (CAN) group has been performing a re-analysis of published photoelectric data and an observational survey to search for $\delta$ Scuti type pulsating components in detached and semi-detached eclipsing binary systems. Recently, they have discovered three new pulsators: R CMa, AS Eri and TW Dra (Mkrtichian et al. 2002a). In Korea, we started a CCD photometric survey for the same purpose from

Send offprint requests to: S.-L. Kim, e-mail: slkim@kao.re.kr
September 2001 onwards and found three new field $\delta$ Scuti variables serendipitously (Kim et al. 2002). Thanks to the high precision CCD photometry and observational survey projects, the number of these interesting objects is expected to increase in the near future.

Y Cam $\left(\mathrm{RA}_{2000}=7^{\mathrm{h}} 41^{\mathrm{m}} 11^{\mathrm{s}} .0, \operatorname{Dec}_{2000}=76^{\circ} 04^{\prime} 26^{\prime \prime}, V=\right.$ $10^{\mathrm{m}} 56, B-V=0.32$, Spectral type $\left.=\mathrm{A} 7 \mathrm{~V}\right)$ is an Algol type eclipsing binary star. Broglia \& Conconi (1984) derived its orbital period of 3.30552340 day and high inclination of about $86^{\circ}$ from $B, V$ photometry. They suggested that the orbital period variation shown in the $(\mathrm{O}-\mathrm{C})$ diagram is resulted from combining an apsidal motion of the binary system and a light time effect by the third body.

Broglia (1973) firstly detected small brightness fluctuations of Y Cam, out of the deepest primary minimum. Considering the spectral type and variable properties, a period of 0.063 day and maximum amplitude of 0.04 , he suggested that the hotter component is a $\delta$ Scuti type pulsator. The pulsating amplitudes changed with observation times but were not correlated with orbital phase, implying that the amplitude variation is not caused by the binary nature (Broglia \& Marin 1974). After ten years, Broglia \& Conconi (1984) detected two very closely separated frequencies from new $B, V$ observations. They proposed that the primary component of Y Cam is a non-radial pulsator.

In this paper, we present CCD photometric results of Y Cam, with emphasis on the pulsational characteristics of the primary component. The observations and data reduction are described in Sect. 2 and the detailed results in Sect. 3. 


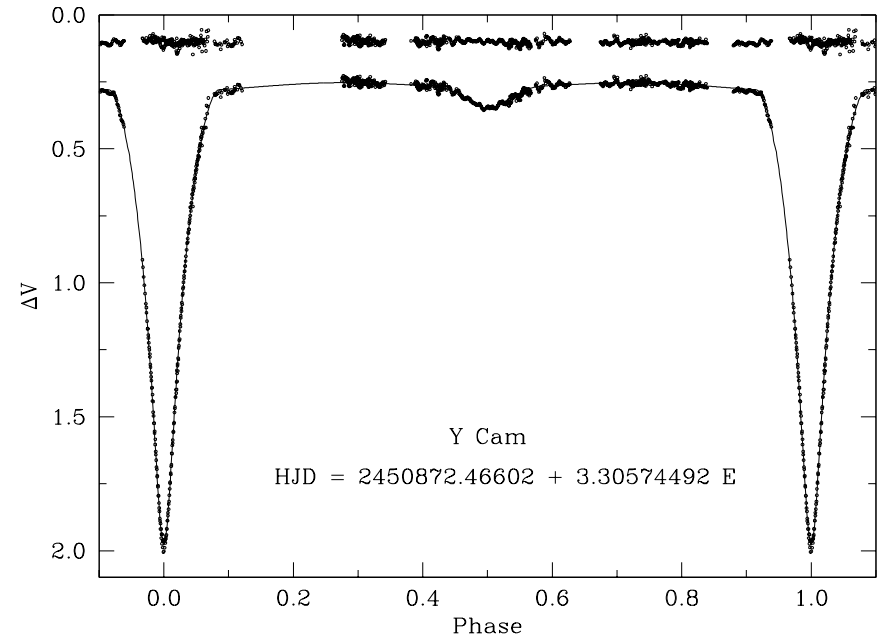

Fig. 1. Phase diagram and residuals (upper) after fitting the photometric solution represented by a solid line. Small amplitude light variations are remarkable, particularly during the secondary minimum phase.

\section{Observation and data reduction}

We carried out CCD photometric observations for 16 nights from November 2000 to May 2001, using a PM512 CCD camera attached to the $61 \mathrm{~cm}$ telescope at the Sobaeksan Optical Astronomy Observatory in Korea. The CCD chip has $512 \times$ 512 pixels and a pixel size of $20 \mu \mathrm{m}$. The field of view (FOV) of a CCD image is about 4.3 34.3 on the f/13.5 Cassegrain focus of the telescope. A nearby star with similar brightness and colour to Y Cam, SAO $6274\left(\mathrm{RA}_{2000}=7^{\mathrm{h}} 42^{\mathrm{m}} 30^{\mathrm{s}} .5, \mathrm{Dec}_{2000}=\right.$ $76^{\circ} 05^{\prime} 04^{\prime \prime}, V=10^{\mathrm{m}} \cdot 27, B-V=0.29$, Spectral type = A5) was selected as a comparison star. It did not show any peculiar light variations during our observing runs as well as the previous observations by Broglia \& Marin (1974) and Broglia \& Conconi (1984). Since the FOV was not large enough to observe two stars simultaneously at the same CCD image, we monitored them alternately.

Using the differential photometry software ADPS (Park 1993; Park \& Lee 1995), the observations were performed automatically and instrumental magnitudes were obtained in realtime. As a result, a total of $1054 \mathrm{~V}$-band differential magnitudes were collected.

We obtained the eclipsing data of two primary minima and one secondary minimum. Orbital phases were calculated from the following equation

Min HJD $=2450872.46602+3.30574492 \times E$.

Because the orbital period of Y Cam has been known to change with time, minimum epoch and orbital period were newly derived from recent data (Borkovits \& Bíró 1998; Nelson 2000; Bíró \& Borkovits 2000). We included two new primary minima, HJD 2451887.3300 and 2452003.0314, obtained in this study. Detailed results of the eclipsing phenomenon such as orbital period variation and photometric solution, etc., will be presented in a separate paper (Lee et al. 2002). Phase diagram and residuals after fitting the photometric solution are shown

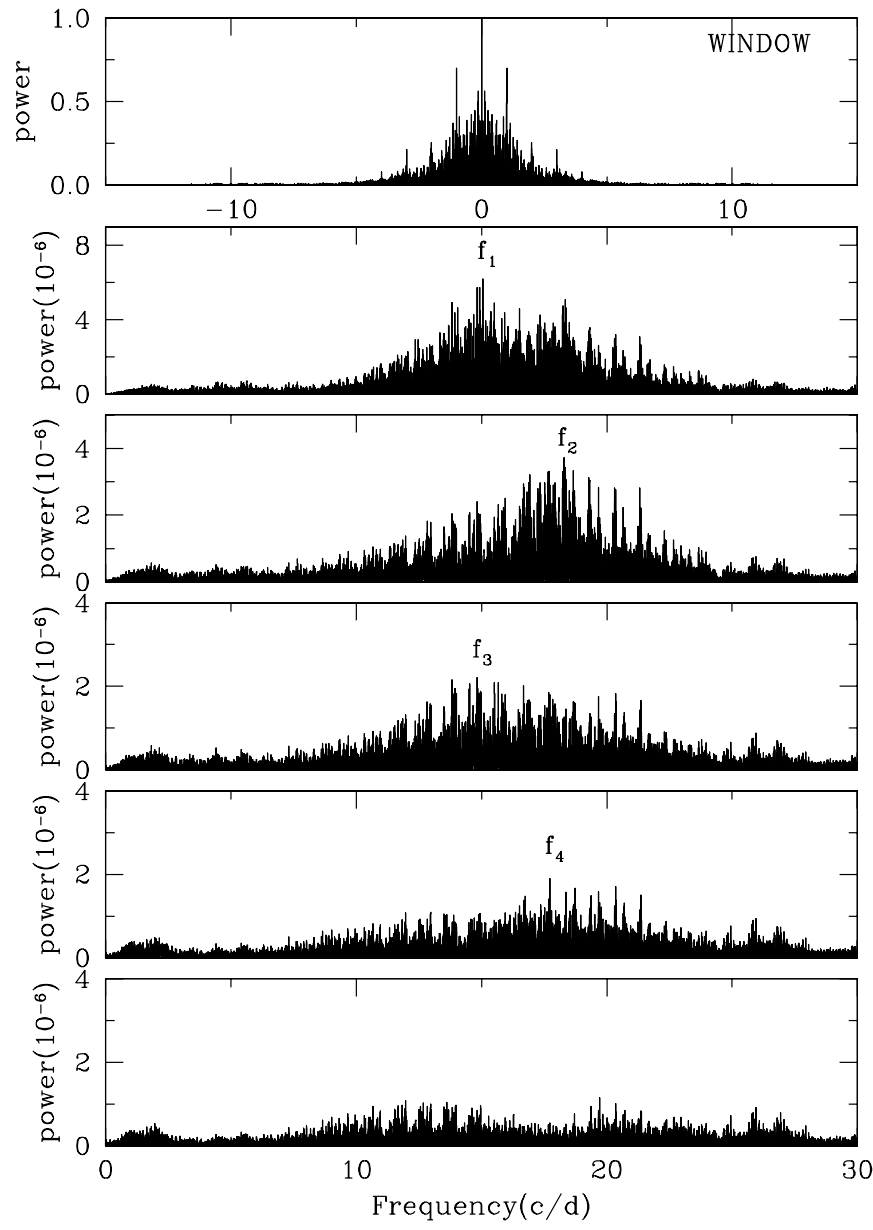

Fig. 2. Power spectra using the residuals except for the primary eclipsing phase. Spectral window is shown in the top panel. Four frequencies can be identified in the next four panels.

in Fig. 1. Small amplitude fluctuations with short period are noticeable, particularly during the secondary minimum phase.

\section{Pulsational characteristics}

\subsection{Multiple frequency analysis}

The residuals show very complicated curves and its amplitudes vary from cycle to cycle, indicating that multiple periods are superimposed. In order to investigate these oscillating features, we applied the multiple frequency analysis using the Discrete Fourier Transform (DFT) and a linear least square fitting method (Kim \& Lee 1996). Since the hotter component of Y Cam is a $\delta$ Scuti type pulsator, we made use of only the data obtained for 12 nights having orbital phases from 0.15 to 0.85 , i.e., excluding the data around the primary minima.

The power spectra are shown in Fig. 2. The spectral window in the first panel shows strong side bands, particularly at 1 cycle/day which is produced by the daily gaps of observation. After the successive prewhitening of each frequency peak in the next four panels, we derived four frequencies of $f_{1}=15.0473 \mathrm{c} / \mathrm{d}, f_{2}=18.2852 \mathrm{c} / \mathrm{d}, f_{3}=14.8203 \mathrm{c} / \mathrm{d}$ and $f_{4}=17.7348 \mathrm{c} / \mathrm{d}$. Some additional peaks still exist in the last panel but their signal to noise amplitude $(S / N)$ ratios are 

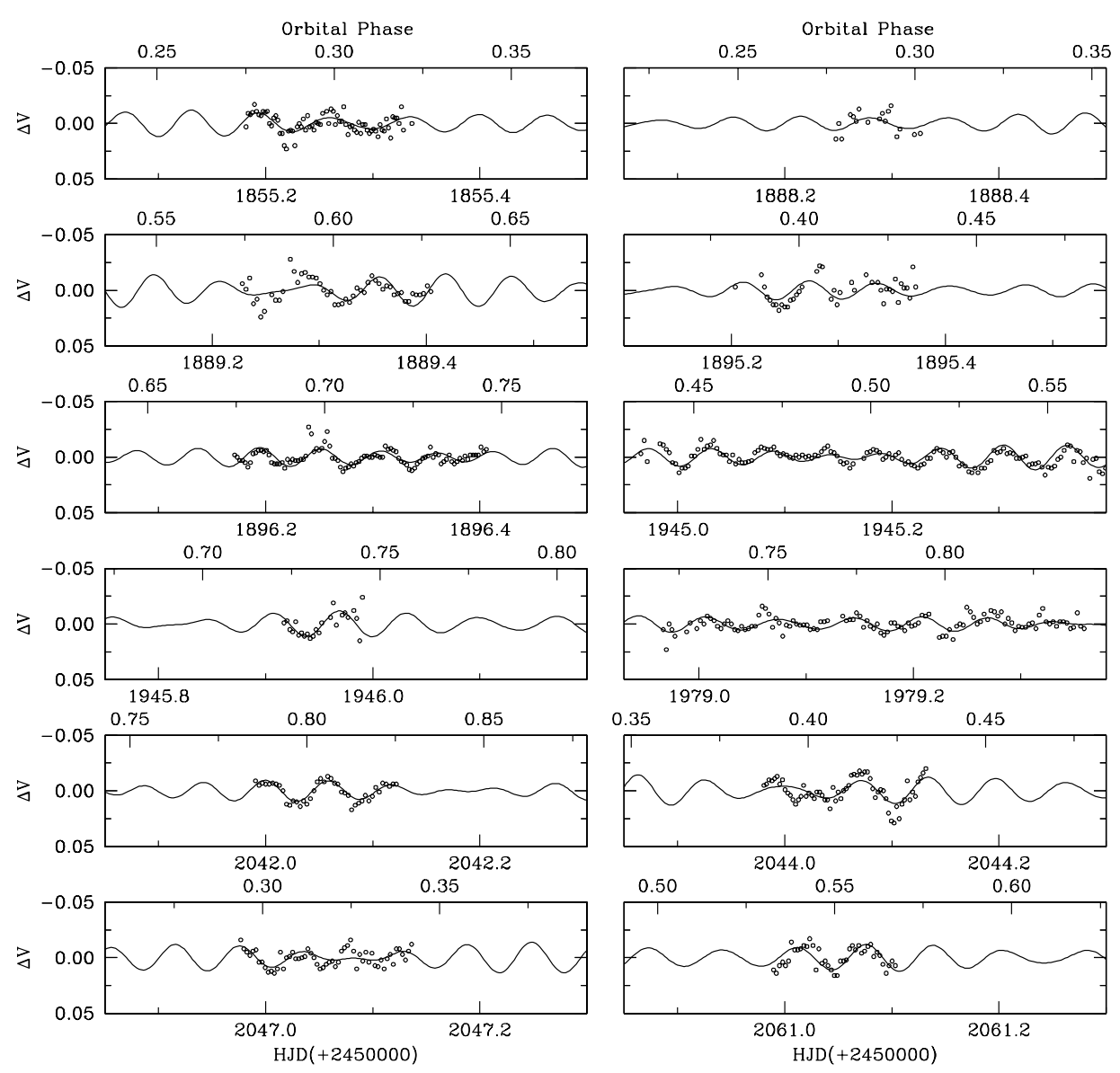

Fig. 3. Light variations of the residuals obtained for 12 nights having orbital phases from 0.15 to 0.85 . Synthetic curves computed from our four frequencies are superimposed.

smaller than 4.0, the empirical criterion proposed by Breger et al. (1993). Detailed results of the analysis are summarized in Table 1, including the previous results by Broglia \& Conconi (1984) for comparison. Figure 3 displays light variations of the residuals and synthetic curves computed from our four frequencies.

In addition to the $S / N$ ratio in the power spectra, standard deviation of the successive prewhitening procedure was examined to find the step at which we should stop detecting frequencies. The idea was recently introduced by Paparo et al. (2002). The deviation decreases as the number of prewhitened frequencies increases, and the decline is steeper at the early step than at the late one (Fig. 4). Slopes of $\Delta$ deviation (difference between each step) against the number of frequencies, represented by solid lines at the lower panel in Fig. 4, are decelerating rates of the deviation. There is a noticeable change near the fifth frequency. This indicates that their sources might be different. We suggest that these are real periodic signals up to the fourth frequency and random fluctuations after the fifth one. This is in good agreement with the results deduced from the $S / N$ ratio.

We examined the residuals around primary eclipsing phase separately because it could give us a clue on mode identification. Figure 5 displays the data obtained for 4 nights of orbital phases from 0.85 to 1.15 . The synthetic curves calculated from the other phase data are superimposed. The data are poorly
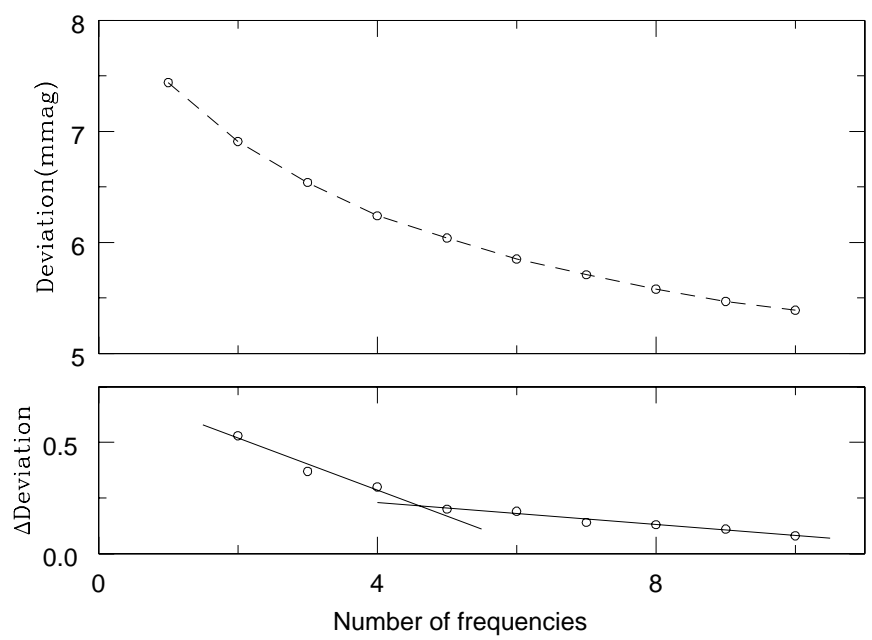

Fig. 4. Standard deviations of the successive prewhitening procedure (upper) and their differences between each step (lower). The decelerating rates represented by solid lines are changed near the fifth step.

matched with the curves, particularly near the deepest minimum phase, showing that amplitudes and pulsation phases are different between two data sets. Power spectra using the residuals are shown in Fig. 6. Four frequencies derived from the other phase data are marked by four vertical solid lines. A low 
Table 1. Multi-frequency analysis of the $\delta$ Scuti type pulsation.

\begin{tabular}{|c|c|c|c|c|c|c|c|}
\hline \multicolumn{4}{|c|}{ This study (Nov. 2000 May 2001) } & \multicolumn{2}{|c|}{ BC84 Group I ( $V$-band) } & \multicolumn{2}{|c|}{ BC84 Group II ( $V$-band) } \\
\hline Frequency & $A_{j}^{\dagger}$ & $S / N^{\ddagger}$ & $\phi_{j}^{\dagger}$ & Frequency & $A_{j}$ & Frequency & $A_{j}$ \\
\hline$f_{1}=15.0473 \mathrm{c} / \mathrm{d}$ & $5.8 \pm .3 \mathrm{mmag}$ & 7.2 & $-0.89 \pm .06$ & $15.0473 \mathrm{c} / \mathrm{d}$ & $12.2 \mathrm{mmag}$ & $15.0473 \mathrm{c} / \mathrm{d}$ & $11.9 \mathrm{mmag}$ \\
\hline$f_{2}=18.2852$ & $3.9 \pm .3$ & 5.6 & $+1.06 \pm .08$ & - & - & - & - \\
\hline$f_{3}=14.8203$ & $3.4 \pm .3$ & 4.3 & $-1.31 \pm .10$ & 14.5890 & 6.0 & 14.6593 & 10.3 \\
\hline$f_{4}=17.7348$ & $2.8 \pm .3$ & 4.0 & $-0.35 \pm .12$ & - & - & - & - \\
\hline Deviations & $6.24 \mathrm{mmag}$ & & & & $6.8 \mathrm{mmag}$ & & $7.9 \mathrm{mmag}$ \\
\hline
\end{tabular}

$\dagger^{\dagger} V=V_{\circ}+\Sigma_{j} A_{j} \cos \left\{2 \pi f_{j}\left(t-t_{\circ}\right)+\phi_{j}\right\}, \quad t_{\circ}=\mathrm{HJD} 2450000.0$.

: $S / N=$ (power for each frequency / mean power in the range of $0 \sim 30 \mathrm{c} / \mathrm{d}$ after prewhitening of all frequencies $)^{1 / 2}$.

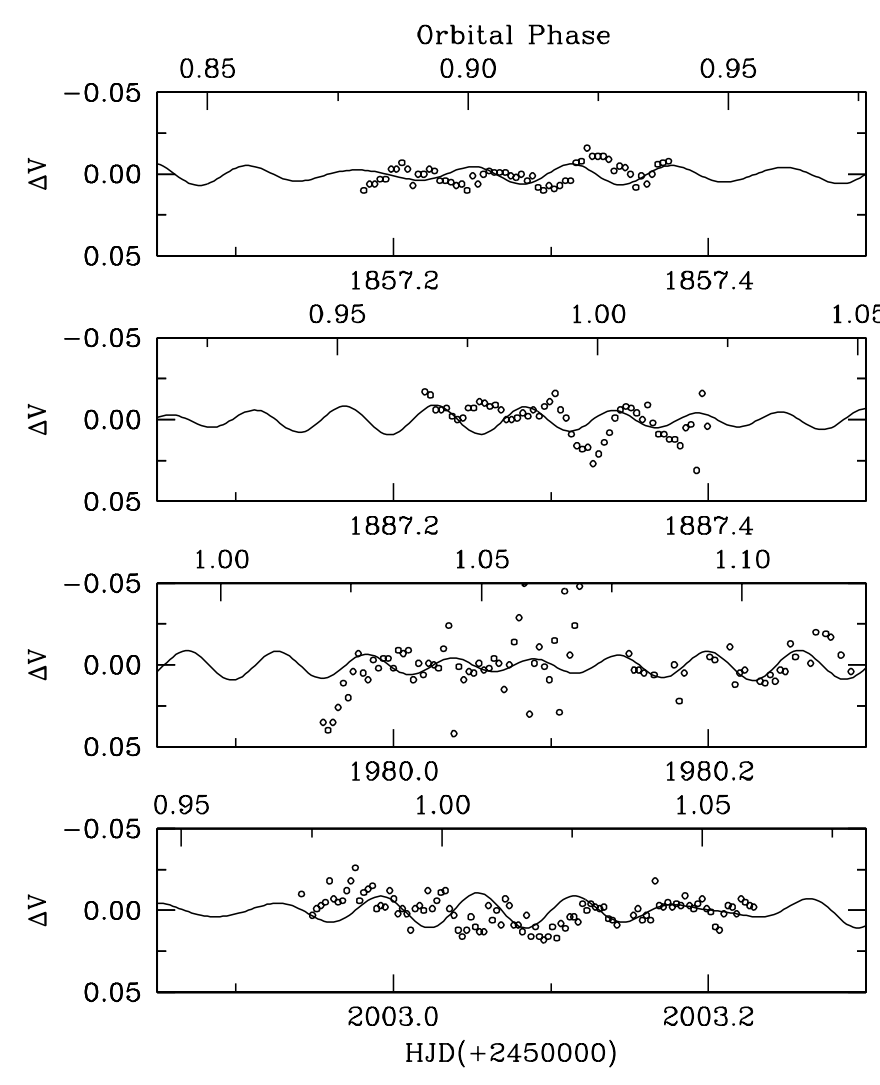

Fig. 5. Residuals around the primary eclipsing phase. Synthetic curves calculated from the other orbital phase data are poorly matched with the data.

frequency at $4.4 \mathrm{c} / \mathrm{d}$ might be originated from the eclipsing light curves. Since these differences of amplitudes, pulsation phases and frequency solutions could be resulted from spatial filtrations of non-radial modes during the primary eclipse (Mkrtichian et al. 2002b), the hotter component of Y Cam should be excited in non-radial pulsation modes. In comparison with the case of Y Cam, pulsating components of AB Cas (Rodríguez et al. 1998) and RZ Cas (Ohshima et al. 2001) seem to be excited in radial modes, showing similar frequency solutions between the primary eclipsing phase data and the others. Further extensive photometric and spectroscopic observations during the primary eclipse of Y Cam would give us more detailed information on non-radial modes.

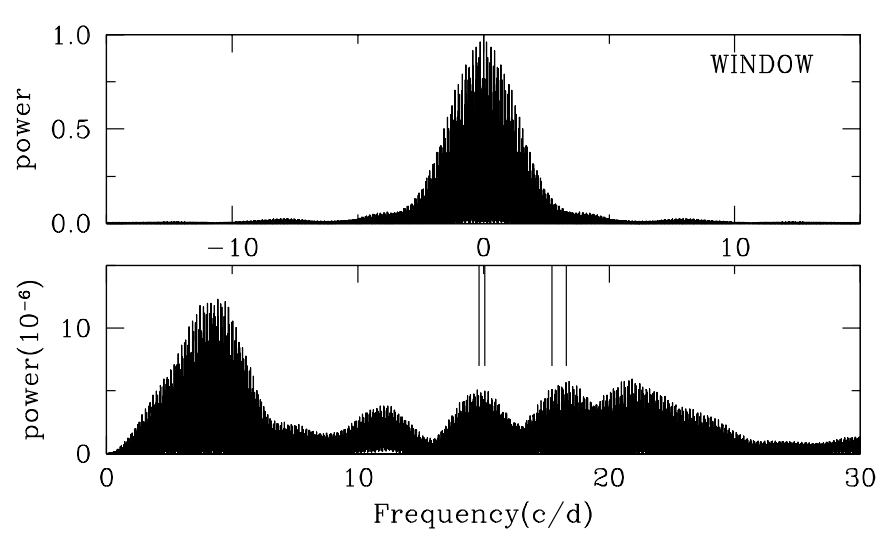

Fig. 6. Power spectra using the residuals around the primary eclipsing phase. Four frequencies derived from the other orbital phase data are marked by four vertical solid lines.

\subsection{Variations of pulsational characteristics}

The first frequency of $15.0473 \mathrm{c} / \mathrm{d}$ turned out to be constant over about 40 years from JD 2437582 to JD 2452061 (Table 1). But the other frequencies have been changed or newly excited. Amplitudes of the residuals were changed from cycle to cycle in our observations but from night to night in the previous ones by Broglia \& Conconi (1984). It supports that the differences between two dominant frequencies are larger in our observations.

It should be noted that our $V$ amplitude of the first frequency decreased to about half the previous values. In order to study whether its pulsation phase was stable, pulsation epochs with maximum brightness were carefully examined. We used the ephemeris for $\delta$ Scuti type pulsation with large amplitude, which was derived by Broglia \& Conconi (1984).

Max HJD $=2437375.458+0.066457537 \times n$.

Maximum epochs, cycle numbers $n$ and $(\mathrm{O}-\mathrm{C})$ values of the epochs are listed in Table 2, including seven new epochs obtained in this study. The other frequencies modulated in light curves could make a little large scatter in the $(\mathrm{O}-\mathrm{C})$ diagram of the first frequency. We chose the maximum epochs with large amplitudes to lessen the scatter.

The amplitudes and $(\mathrm{O}-\mathrm{C})$ variations of the first frequency are shown in Fig. 7. Our $(\mathrm{O}-\mathrm{C})$ values were significantly 
Table 2. Maximum epochs of the $\delta$ Scuti type pulsation.

\begin{tabular}{|c|c|c|c|}
\hline Max HJD & $n$ & $(\mathrm{O}-\mathrm{C})$ & Reference \\
\hline 2437375.455 & 0 & -0.003 & BC84 \\
\hline 2437380.376 & 74 & $+0 \mathrm{~d} 000$ & $\mathrm{BC} 84$ \\
\hline 2437382.306 & 103 & +0.003 & BC84 \\
\hline 2437398.388 & 345 & +0.002 & BC84 \\
\hline 2437608.391 & 3505 & -0.001 & BC84 \\
\hline 2437641.286 & 4000 & -0.002 & $\mathrm{BC} 84$ \\
\hline 2437696.313 & 4828 & -0.002 & BC84 \\
\hline 2437699.307 & 4873 & +0.001 & BC84 \\
\hline 2437757.322 & 5746 & -0.001 & $\mathrm{BC} 84$ \\
\hline 2437760.370 & 5792 & -0.010 & BC84 \\
\hline 2437764.369 & 5852 & $+0 \mathrm{~d} 001$ & $\mathrm{BC} 84$ \\
\hline 2437765.493 & 5869 & -0.004 & BC84 \\
\hline 2437785.505 & 6170 & +0.004 & $\mathrm{BC} 84$ \\
\hline 2437998.502 & 9375 & +0.005 & BC84 \\
\hline 2438053.319 & 10200 & -0.006 & BC84 \\
\hline 2438314.375 & 14128 & $+0 \mathrm{~d} 005$ & BC84 \\
\hline 2438400.309 & 15421 & +0.009 & $\mathrm{BC} 84$ \\
\hline 2438403.285 & 15466 & $-0 \mathrm{~d} 005$ & $\mathrm{BC} 84$ \\
\hline 2438760.303 & 20838 & +0.003 & BC84 \\
\hline 2438765.290 & 20913 & +0.006 & $\mathrm{BC} 84$ \\
\hline 2444222.178 & 103024 & -0.001 & BC84 \\
\hline 2444224.176 & 103054 & +0.003 & $\mathrm{BC} 84$ \\
\hline 2444901.439 & 113245 & -0.003 & BC84 \\
\hline 2444911.403 & 113395 & -0.007 & $\mathrm{BC} 84$ \\
\hline 2444938.396 & 113801 & $+0 \mathrm{~d} 004$ & $\mathrm{BC} 84$ \\
\hline 2444940.250 & 113829 & -0.003 & BC84 \\
\hline 2444999.395 & 114719 & -0.005 & $\mathrm{BC} 84$ \\
\hline 2445028.378 & 115155 & +0.002 & BC84 \\
\hline 2445370.300 & 120300 & +0.000 & BC84 \\
\hline 2445383.397 & 120497 & +0.005 & BC84 \\
\hline 2451855.194 & $217879(80)$ & $+0.034(-0.032)$ & Ours \\
\hline 2451889.352 & 218393(94) & $+0.033(-0.033)$ & Ours \\
\hline 2451896.195 & $218496(97)$ & $+0.031(-0.035)$ & Ours \\
\hline 2451945.306 & $219235(36)$ & $+0.030(-0.037)$ & Ours \\
\hline 2452042.002 & $220690(91)$ & $+0.030(-0.036)$ & Ours \\
\hline 2452044.070 & $220721(22)$ & $+0{ }^{\mathrm{d}} 038(-0.028)$ & Ours \\
\hline 2452061.075 & $220977(78)$ & $+0.030(-0.037)$ & Ours \\
\hline
\end{tabular}

different from the previous ones. It could be caused by changing the first frequency itself. We can fit three clustering data with a period changing rate of $(1 / P) \mathrm{d} P / \mathrm{d} t= \pm 1.2 \times 10^{-8}$ year $^{-1}$ (dashed lines). The rate matches well with observed and theoretically predicted values of $\delta$ Scuti stars (Breger 2000a). A sudden jump of pulsation phase (solid lines) can also explain this difference. As in the case of a well-known $\delta$ Scuti star 4 CVn (Breger 2000b), the amplitude decreased considerably and the phase shifted to about half a pulsating cycle. At present, because of having only three data sets, we could not make a decision whether the $(\mathrm{O}-\mathrm{C})$ variations resulted from a period change or a sudden jump of pulsation phase. Further observations are needed to monitor these variations.

\section{Summary}

New photometric observations of the eclipsing binary Y Cam were performed for 16 nights from November 2000 to

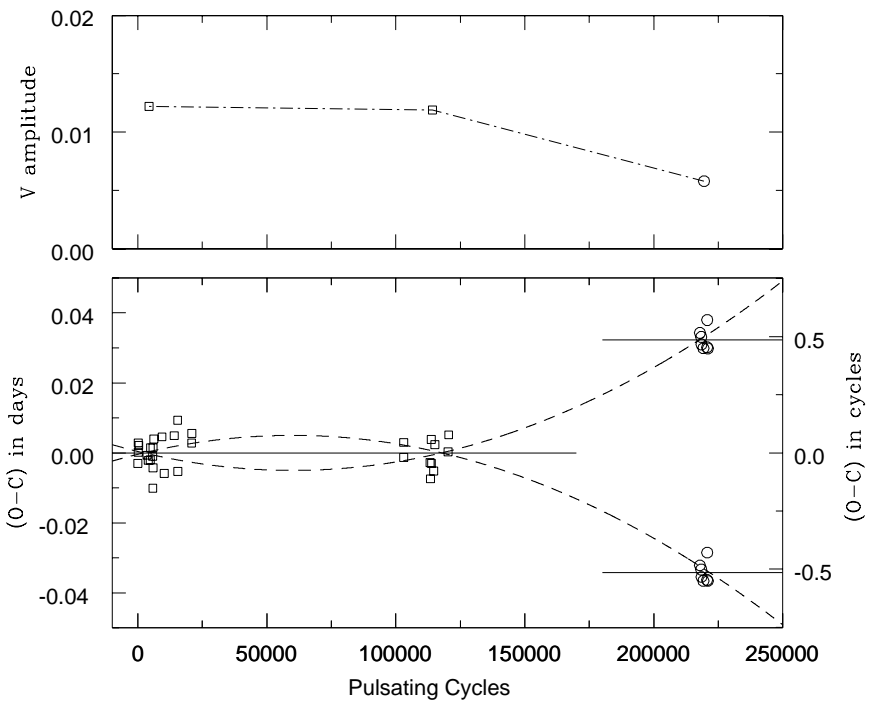

Fig. 7. $V$ amplitudes and (O-C) values of the first pulsating frequency. Our data are represented by open circles. The $(\mathrm{O}-\mathrm{C})$ variations can be fitted by a period change with the rate of $(1 / P) \mathrm{d} P / \mathrm{d} t= \pm 1.2 \times$ $10^{-8}$ year $^{-1}$ (dashed lines) as well as a sudden phase jump about half a pulsating cycle (solid lines).

May 2001. After removing the eclipsing light curves, we obtained the following interesting results on pulsational properties of its primary component in comparison with the previous data.

(i) From the multiple frequency analysis, four pulsation frequencies of $15.0473 \mathrm{c} / \mathrm{d}, 18.2852 \mathrm{c} / \mathrm{d}, 14.8203 \mathrm{c} / \mathrm{d}$ and $17.7348 \mathrm{c} / \mathrm{d}$ were derived. The first frequency remained nearly constant over 40 years but the other frequencies were changed or newly excited. Our $V$ amplitude of the first frequency decreased to about a half.

(ii) Our $(\mathrm{O}-\mathrm{C})$ values of the first frequency shifted to about half a pulsation cycle. At present, it is difficult to decide whether this difference is caused by a period change or by a sudden jump of pulsation phase.

(iii) Light variations around the primary eclipsing phases were much different from those of the other phases. We presented a possibility that non-radial pulsation modes of the hotter components in eclipsing binary systems could be identified from spatial filtrations during the primary eclipse.

(iv) In addition to the signal to noise amplitude ratio, standard deviation of the successive prewhitening procedure might be useful as a criterion to stop detecting multiple frequencies. The idea was recently introduced by Paparo et al. (2002).

Acknowledgements. We thank Dr. Y.-J. Moon and Dr. H.-K. Moon for their careful reading and comments. This research made use of the SIMBAD database, operated at CDS, Strasbourg, France. This work was supported by Korea Research Foundation Grant (KRF-2000-015DP0444).

\section{References}

Bíró, I. B., \& Borkovits, T. 2000, Inf. Bull. Variable Stars, No. 4967 Borkovits, T., \& Bíró, I. B. 1998, Inf. Bull. Variable Stars, No. 4633 Breger, M. 2000a, MNRAS, 313, 129 
Breger, M. 2000b, in Delta Scuti and related stars, ed. M. Breger, \& M. H. Montgomery, PASPC, 210, 3

Breger, M., Stich, J., Garrido, R., et al. 1993, A\&A, 271, 482

Broglia, P. 1973, Inf. Bull. Variable Stars, No. 823

Broglia, P., \& Marin, F. 1974, A\&A, 34, 89

Broglia, P., \& Conconi, P. 1984, A\&A, 138, 443 (BC84)

Kim, S.-L., \& Lee, S.-W. 1996, A\&A, 310, 831

Kim, S.-L., Kwon, S.-G., Youn, J.-H., Kyeong, J.-M., \& Lee, J. W. 2002, Inf. Bull. Variable Stars, No. 5244

Lampens, P., \& Boffin, H. M. J. 2000, in Delta Scuti and related stars, ed. M. Breger, \& M. H. Montgomery, PASPC, 210, 309

Lee, J. W., Kim, S.-L., Youn, J.-H., Kwon, S.-G., \& Kim, C. H. 2002, in preparation (in Korean)

Mkrtichian, D. E., Kusakin, A. V., Gamarova, A. Yu., et al. 2002a, in Observational aspects of pulsating B \& A stars, ed. C. Sterken, \& D. W. Kurtz, PASPC, 256, 259
Mkrtichian, D. E., Kusakin, A. V., Gamarova, A. Yu., \& Nazarenko, V. 2002b, in Radial and nonradial pulsations as probes of stellar physics, ed. C. Aerts, T. R. Bedding, \& J. Christensen-Dalsgaard, PASPC, 259, 96

Nelson, R. H. 2000, Inf. Bull. Variable Stars, No. 4840

Ohshima, O., Narusawa, S.-Y., Akazawa, H., et al. 2001, AJ, 122, 418

Park, N.-K. 1993, Publications of the Korean Astronomical Society, 8, 185

Park, N.-K., \& Lee, S.-W. 1995, AJ, 109, 774

Paparo, M., Shibahashi, H., \& Sterken, C. 2002, in Radial and nonradial pulsations as probes of stellar physics, ed. C. Aerts, T. R. Bedding, \& J. Christensen-Dalsgaard, PASPC, 259, 94

Rodríguez, E., Claret, A., Sedano, J. L., García, J. M., \& Garrido, R. 1998, A\&A, 340, 196 